2006-11-01

\title{
Experimental Investigation on VolP Performance and the Resource Utilization in 802.11b WLANs
}

\author{
Miroslaw Narbutt \\ Technological University Dublin, miroslaw.narbutt@tudublin.ie \\ Mark Davis \\ Technological University Dublin, mark.davis@tudublin.ie
}

Follow this and additional works at: https://arrow.tudublin.ie/commcon

Part of the Electrical and Electronics Commons

\section{Recommended Citation}

Narbutt, M. \& Davis, M. (2006) Experimental investigation on VolP performance and the resource utilization in 802.11b WLANs. IEEE Conference on Local Computer Networks (LCN'06), November, 2006, Tampa

This Conference Paper is brought to you for free and open access by the Communications Network Research Institute at ARROW@TU Dublin. It has been accepted for inclusion in Conference papers by an authorized administrator of ARROW@TU Dublin. For more information, please contact arrow.admin@tudublin.ie, aisling.coyne@tudublin.ie,gerard.connolly@tudublin.ie.

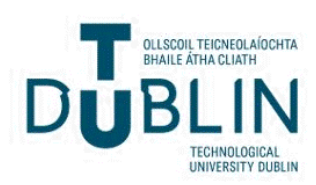




\title{
Experimental investigation on VoIP performance and the resource utilization in 802.11b WLANs
}

\author{
Miroslaw Narbutt and Mark Davis \\ Communications Network Research Institute \\ School of Electronic and Communications Engineering, \\ Dublin Institute of Technology, IRELAND \\ narbutt@cnri.dit.ie,mark.davis@dit.ie
}

\begin{abstract}
In a shared medium network like the $802.11 \mathrm{~b}$ WLAN, predicting the quality of VoIP calls from the resource usage of the wireless medium is highly desirable. Analyzing the bandwidth usage at the L2/MAC layer may be especially useful for potential QoS provisioning and call admission schemes. This paper experimentally investigates the relationship between resource utilization in WLANs and the quality of VoIP calls transmitted over wireless medium. Specifically we evaluate how the amount of free bandwidth influences transmission impairments (i.e. delay, loss, and jitter) and thus call quality. Resource utilization (under the MAC bandwidth components framework) is calculated by a WLAN resource monitoring application that passively "sniffs" packets at the L2/MAC layer and analyses their headers and temporal characteristics. The quality of VoIP calls is predicted using an extended version of the ITU-T Emodel, which estimates user satisfaction from time varying transmission impairments.
\end{abstract}

\section{Introduction}

IEEE 802.11 compliant wireless networks (WLANs) use a media access control (MAC) mechanism, namely Carrier Sense Multiple Access with Collision Avoidance (CSMA/CA), which requires the wireless stations to compete with one another in order to gain access to the wireless medium. In $802.11 \mathrm{~b}$ networks all stations contending for access to the medium do so with the same priority. A direct consequence of this competition is that heavy or greedy users can have a detrimental effect on the overall performance of the network by essentially depriving other users of bandwidth. A more subtle consequence is that the capacity of the network is not fixed and varies with the level and nature of the transmitted load. As a result, different stations will experience different capacities depending on the size and sending rate of the packets comprising the load of the station itself and the other contending stations.

Due to the large overhead involved in transmitting small packets, the bandwidth available for VoIP traffic is far less than the bandwidth available for data traffic. This overhead comprises the additional time required to send packet headers introduced by various networking layers and the access time (in terms of the backoff and deferral times) introduced by the MAC mechanism. The overhead introduced by the MAC mechanism represents the cost of accessing the medium which is not fixed but increases as the number of contending stations increases owing to an increased number of deferrals.

Currently available WLAN protocol analyzers operate at layer 3 and above. They are capable of measuring the WLAN bandwidth consumed by a station in transmitting its load (the load bandwidth), which is directly related to the throughput of the station. However, these layer 3 WLAN protocol analyzers are not able to measure the bandwidth cost (the access bandwidth) associated with the time a station spends attempting to access the medium in order to transmit its load. Consequently, these analyzers cannot measure the bandwidth usage at the L2/MAC layer which makes performance monitoring and servicing provisioning on WLANs difficult to realize.

The WLAN Resource Monitor described in [1,2] overcomes these difficulties by directly measuring various aspects of WLAN operation such as contention for access, bandwidth utilization, capacity at the L2/MAC layer of the wireless medium.

In this paper we experimentally study the relationship between bandwidth utilization in the wireless LAN and the quality of VoIP calls transmitted over the wireless medium. Specifically we evaluate 
how the overall capacity is shared between three basic MAC bandwidth components (i.e. the load, access, and free components) under different traffic load as the number of VoIP calls increases and how it influences transmission impairments (i.e. delay, loss, and jitter) and thus degrades call quality. We believe that this type of resource information could be useful for potential QoS provisioning and call admission schemes. This paper is structured as follows: In Section 2 we describe the concept of three interrelated MAC bandwidth components. In Section 3 we introduce a method for predicting VoIP call quality based on transmission impairments. In Section 4 we show the results of our experiments and we discuss the relationship between bandwidth utilization and the quality of VoIP calls transmitted over the wireless medium. Finally, in Section 5, we present our conclusions.

\section{Resource utilization monitoring in 802.11WLAN}

It is possible to identify two basic time intervals on the wireless medium: busy and idle. The busy intervals represent the time during which wireless devices transmit data/managements frames and their positive acknowledgments. The complementary intervals are idle intervals where the wireless medium is silent. These idle intervals are fundamental to the operation of the 802.11 MAC protocol. Wireless devices with a frame to transmit use these idle intervals in order to win transmission opportunities for the frame. Typically the time required to win a transmission opportunity, i.e. the access time, comprises two components: a time spent deferring for DIFS following the medium becoming idle and a time spent decrementing its backoff counter. When a wireless device does not have a frame to transmit, then that idle interval represents unused or free time on the medium. This free time constitutes spare capacity on the medium in the sense that it can be utilized to win additional transmission opportunities for the device if required.

Figure 1 shows these various time intervals of interest. $T_{\text {busy }}(i)$ are the busy time intervals on the medium when the wireless devices are transmitting their frames, $T_{\text {idle }}(i)$ are the times when the medium is not busy and comprises access time intervals $T_{\text {access }}(i)$ when a device actively contends for transmission opportunities and $T_{\text {free }}(i)$ which represents the unused idle time (i.e. available capacity).

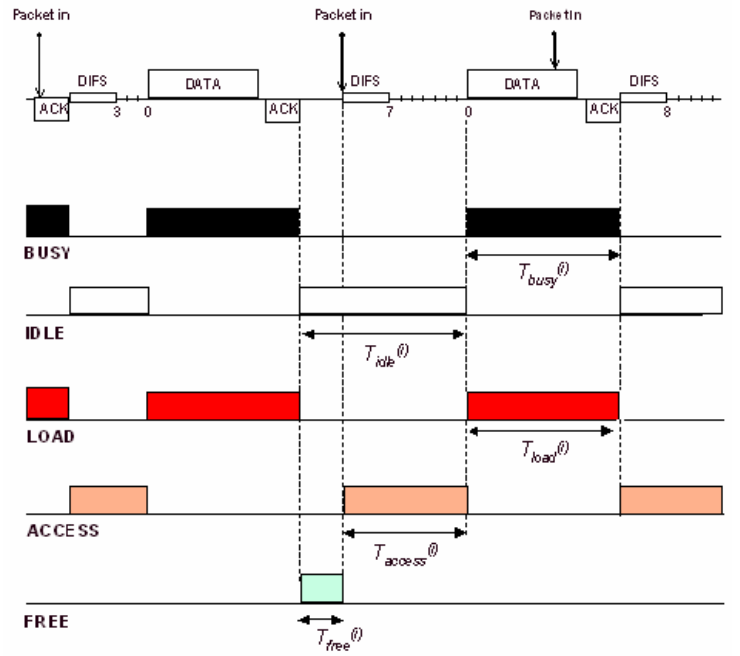

Figure 1. Time intervals involved in accessing the medium

The time intervals involved in accessing the wireless medium are closely related to the MAC bandwidth components we are introducing to characterize WLAN resource utilization. In particular, the bandwidth utilization of a WLAN station is characterized in terms of three interrelated MAC bandwidth components:

- a load bandwidth ( $\left.B W_{\text {load }}\right)$ which is a measure of the WLAN bandwidth consumed by a station in transmitting its load, which is directly related to the throughput of the station;

- an access bandwidth ( $\left.B W_{\text {access }}\right)$ which is a measure of the bandwidth cost associated with accessing the medium in order to transmit its load. The access bandwidth is essentially an overhead requirement imposed on the station;

- a free bandwidth $\left(B W_{\text {free }}\right)$ which is a measure of the unused bandwidth available to the station and represents the spare or available capacity directly related to QoS offered to the station (see Figure 2).

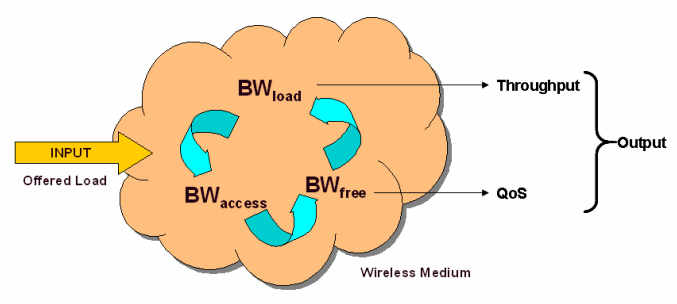

Figure 2. Basic MAC bandwidth components 
A WLAN resource monitoring application based upon this MAC bandwidth components framework is described in $[1,2]$. This monitoring application passively "sniffs" packets at the L2/MAC layer of the wireless medium and analyses their headers and temporal characteristics. It non-intrusively measures both the utilization and availability of bandwidth in real time and on a per-station basis. The ability to measure the bandwidth availability or capacity experienced by individual stations can be particularly useful in realising call admission and QoS provisioning schemes for wireless services.

\section{Predicting VoIP call quality}

A technique that can be used to predict user satisfaction of a conversational speech quality is the ITU-T E-model. The E-Model was originally developed by ETSI [3] as a transmission planning tool, and then standardized by the ITU as G.107 [4] and suggested by TIA [5] as "a tool that can estimate the end-to-end voice quality, taking the IP telephony parameters and impairments into account". This method combines individual impairments (loss, delay, echo, codec type, noise, etc.) due to both the signal's properties and the network characteristics into a single $R$-rating:

$$
R=\left(R_{o}-I_{s}\right)-I_{d}-I_{e}+A
$$

High values of $R$ in a range of $R>90$ should be interpreted as excellent quality, while a lower value of $R$ indicates a lower quality. Values below 50 are clearly unacceptable. In the context of this work, delay impairment $I_{d}$ (which captures the effect of delay and echo) and equipment impairment $I_{e}$ (which captures the effect of information loss caused by the encoding scheme and packet loss) are the most interesting. Because other impairments - such as loud connection and quantization impairment $I_{s}$, the basic signal-tonoise ratio $R_{0}$ (equals 93.2 in narrowband 300 to 3400 $\mathrm{Hz}$ telephony) and the "advantage factor" $A$ (user willingness to accept some quality degradation in return for ease of access) are irrelevant for assessing speech-transmission quality, we can reduce the expression for the $R$ rating to:

$$
R=93.2-I_{d}-I_{e}
$$

Based on the $R$ rating, the ITU-T recommendation G.109 also introduces categories of speech transmission quality and corresponding user satisfaction [6]. Table 1 defines those categories in terms of ranges of $R$.
Table 1. Definition of categories of user satisfaction [6]

\begin{tabular}{|c|c|c|}
\hline $\mathbf{R}$ & MOS & User satisfaction \\
\hline $90-93.2$ & $4.34-4.50$ & very satisfied \\
\hline $80-90$ & $4.03-4.34$ & satisfied \\
\hline $70-80$ & $3.60-4.03$ & some users dissatisfied \\
\hline $60-70$ & $3.10-3.60$ & many users dissatisfied \\
\hline $50-60$ & $2.58-3.10$ & $\begin{array}{c}\text { nearly all users } \\
\text { dissatisfied }\end{array}$ \\
\hline $0-50$ & $1.00-2.58$ & not recommended \\
\hline
\end{tabular}

Using equation (2), and the categories of user satisfaction defined in Table 1, it is possible to draw contours of quality as a function of mouth-to-ear delay (assuming given echo level) and the packet-loss ratio (assuming a given encoding scheme). Such quality contours determine the rating factor $\mathrm{R}$ for all possible combinations of loss and delay with their shape being determined by both impairments $I_{d}$ and $I_{e}$. They give a measure of the impact of packet loss and compression scheme on speech quality and the effect of delay and echo on interactive conversations. Figure 3 shows the quality contours for the G.711 encoding scheme (assuming bursty loss of packets) and for five different echo-loss levels: $T E L R=45,50,55,60,65 d B$ (TELR is the "Talker Echo Loudness Rating", which characterizes the level difference between the original voice and the received echo signal). As can be seen, tolerable mouth-to-ear delay depends strongly on echo cancellation. Moreover, of particular interest here is the ability to find different combinations of loss and delay that result in the same user satisfaction.

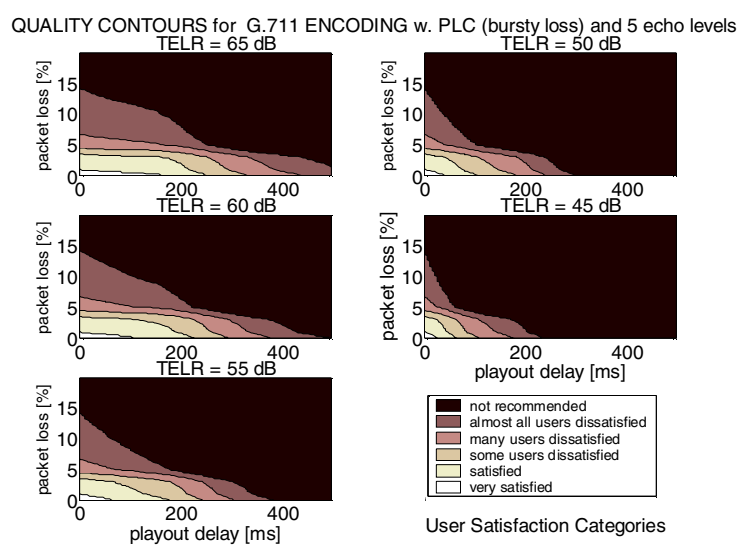

Figure 3. Quality contours for conversational speech for different echo levels (calculated for G.711 w. PLC and bursty loss)

Quality contours are a crucial part of assessing overall user satisfaction as they give a measure of the impact of packet loss and compression scheme on 
speech quality and the effect of delay and echo on interactive conversations.

We first use a playout buffer module to calculate the playout delays and resulting packet loss for a given time interval (for example, 10 seconds). We can then map these playout delays and packet losses on a lossdelay plane that already has quality contours on it (Figure 4). Overall user satisfaction can be obtained from a pie chart that is directly related to distribution of playout delays on quality contours.

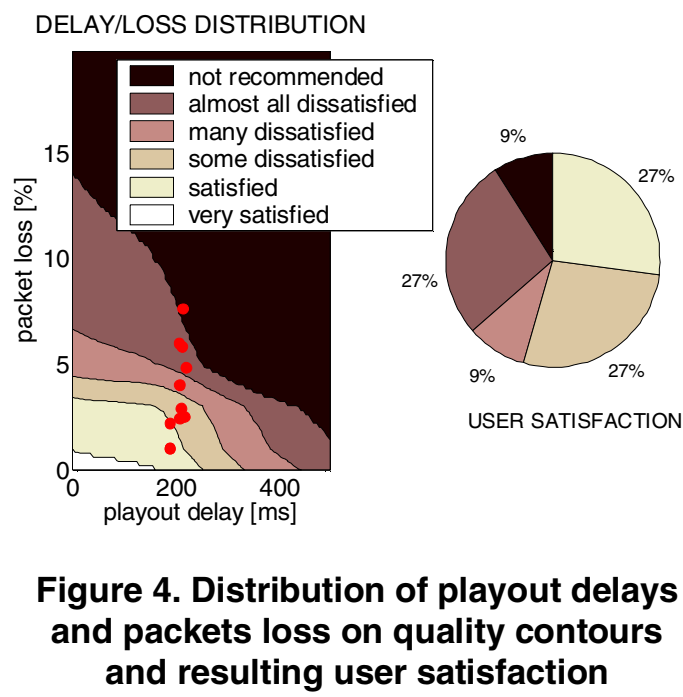

This method of predicting user satisfaction from time varying transmission impairments has shown to be particularly effective in evaluating various playout buffer algorithms [7, 8] and assessing VoIP performance in Voice over WLAN systems $[9,10,11]$. Currently, the E-model based quality contours for predicting speech transmission quality and user satisfaction are being standardized by the ITU-T [12]

\section{Experimental results}

The $802.11 \mathrm{~b}$ wireless/wired testbed consists of 15 desktop PCs acting as wireless VoIP terminals, one desktop PC acting as a background traffic generator, and one desktop PC acting as an access point (AP). All machines in the testbed use the $802.11 \mathrm{~b}$ PCMCIA wireless cards based on Atheros chipsets controlled by MadWiFi wireless drivers and Linux OS (kernel 2.6.9). All of the nodes are also equipped with a $100 \mathrm{Mbps}$ Ethernet cards. The PC that acts as an access point routes traffic between the wired network and the wireless clients, and vice versa (each PC has two interfaces: one on the wireless and one on wired subnet). During the experiments each VoIP terminal runs one VoIP session and all sessions are bi- directional. In this way each terminal acts as the source of an uplink flow and the sink of a downlink for a VoIP session. The wired interface is used to generate background traffic which is routed via the AP to the wireless interface of the same PC. All generated traffic involved a wired and a wireless interface so that no traffic was generated between wireless interfaces. The wireless stations were located within 5 meters range from the AP to ensure that the wireless link quality is good. This testbed is illustrated in Figure 5. Voice traffic was generated using RTPtools [13] which generated G.711 encoded voice packets (80bytes audio frames created every $10 \mathrm{~ms}$ ) with fixed IP packet overhead of 12 bytes for RTP, 8bytes for UDP, and 20bytes for IP layer. During the experiments bidirectional transmission of packets was realized in the form of alternating active and passive periods modeled as four states Markov chain (talker A active, talker B active, both active, both silent). The duration of states and the transitions between them followed the ITU-T recommendation P.59. [14]. This resulted in an ONOFF modulated CBR traffic stream being generated.

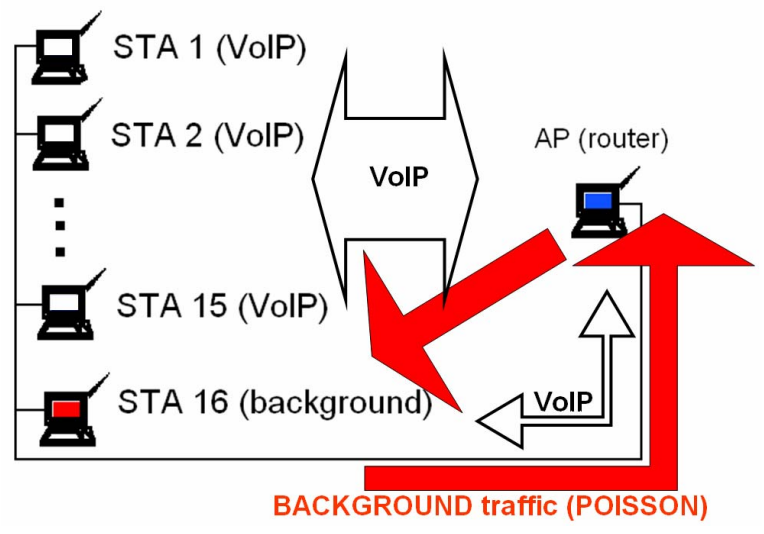

Figure 5. Experimental 802.11b testbed

Background traffic in the form of Poisson distributed UDP packet flow was generated using MGEN traffic generator [15]. For the experiments we used four versions of $2 \mathrm{Mbps}$ traffic load regarding the size and sending rate of the IP packets comprising the load:

- 256 bytes@ 976 pps

- 512 bytes@ 488 pps

- 1024 bytes@244 pps

- 1500 bytes@167pps

During one hour experiments all experimental data (packet arrival times, timestamps, sequence numbers, and marker bits) was collected at the receiving terminal to be processed later (off-line) with a program that 
simulated the behavior of the de-jittering buffer. For the playout buffer module we have chosen the basic adaptive playout algorithm [16]. The main objective of the experiments was to evaluate how overall capacity of the wireless medium is shared between three basic MAC bandwidth components (i.e. the load, access, and free components) as the number of VoIP calls increases and how it influences VoIP call quality under given backround traffic load. For this reason, every hour new calls were successively added to the ongoing calls with the presence of a chosen background traffic.

The bandwidth utilization (in the form of three MAC bandwidth components) was measured during the experiments by the WLAN probe application as it was described in Section 2. The quality of VoIP calls was predicted after each experiment based on transmission impairments as described in Section 3.

Figure 6 shows how the overall capacity of the wireless medium was shared between the three basic MAC bandwidth components during one hour experiment when 14 VoIP simultaneous calls and 2Mbps background traffic (1500bytes IP packets at 167 pps) were carried in the network.

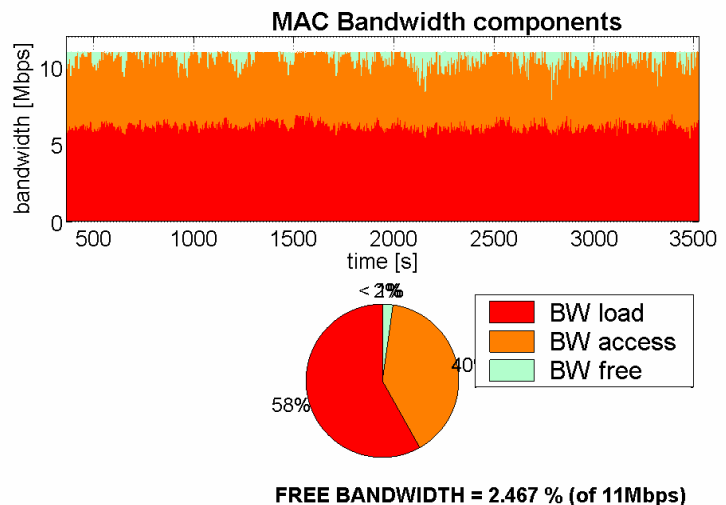

Figure 6. MAC bandwidth utilization with 14 VolP calls and 2Mbps background traffic (1500bytes IP packets@ @ 167pps)

Figure 7 shows how it influenced transmission impairments (delay, loss, and jitter) and thus call quality and overall user satisfaction. With an available free bandwidth of $2.5 \%$ out of $11 \mathrm{Mbps}$, playout delays are below $30 \mathrm{~ms}$ (i.e. mouth-to-ear including packetization at the sender and buffering at the receiver) and packet loss below 5\% with sporadical late packet loss larger than $10 \%$ ( 4 occurences) due to ineffective de-jitter buffering. In this case an average user would be satisfied $91 \%$ of the time, some users could be dissatisfied $2 \%$ of the time, and many users would be dissatisfied $2 \%$ of the time.
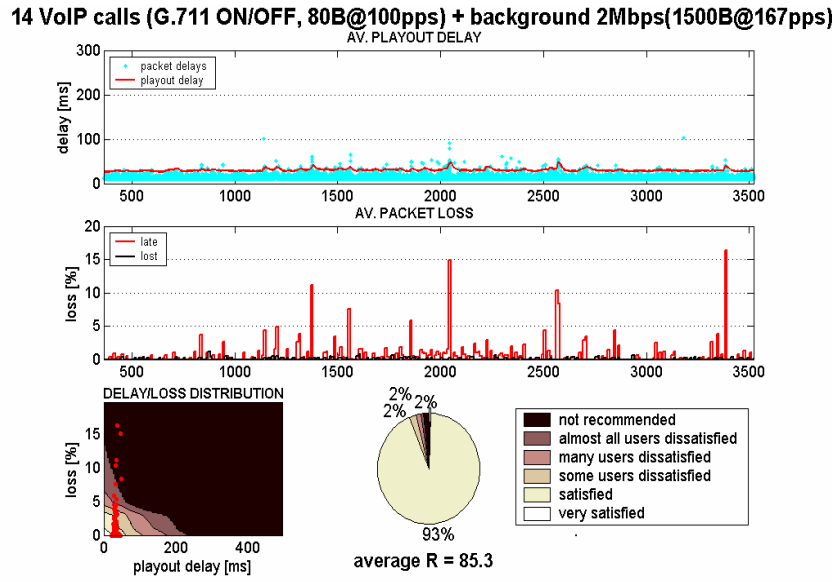

Figure 7. Call quality and user satisfaction with 14 VolP calls and 2Mbps background traffic (1500bytes IP packets @ 167pps)

Figure 8 shows how the overall capacity of the wireless medium was shared between the three basic MAC bandwidth components when the same number of VoIP calls were carried in the network. This time however the size and the sending rate of the packets comprising the backround load is different (1500bytes IP packets at $167 \mathrm{pps}$ ) which results with in no free bandwidth being available. Consequently, network delays occasionally increase up to $200 \mathrm{~ms}$ and large delay variation causes frequent and substantial packet loss (up to 20\%) due to late packet arrival. In this case, an average user would be satisfied only $14 \%$ of the time, some users could be dissatisfied $38 \%$ of the time, many users would be dissatisfied $18 \%$ of the time, almost all users would be dissatisfied $7 \%$ of the time, during $23 \%$ of the time quality was not acceptable.

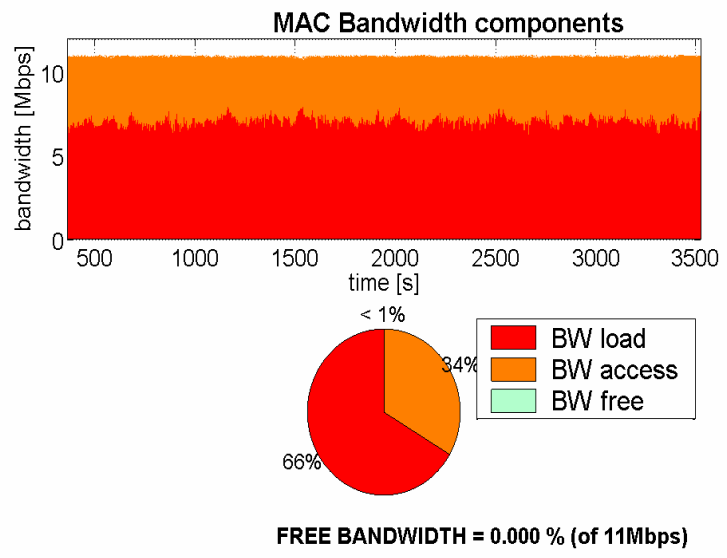

Figure 8. MAC bandwidth utilization with 14 VolP calls and 2Mbps background traffic (512bytes IP packets @ 488pps) 
14 VolP calls (G.711 ON/OFF, 80B@100pps) + background 2Mbps (512B@488pps)
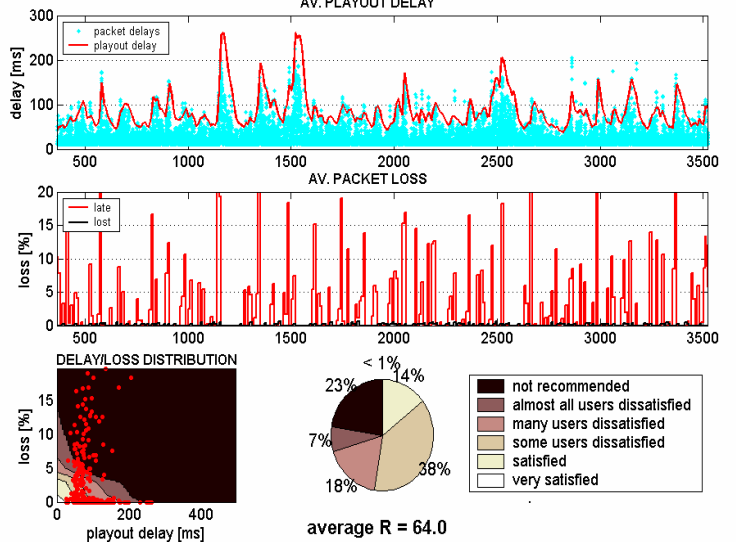

not recommended
almost all users dissatisfied many users dissatisfied some users dissatisfied satisfied

average $R=64.0$

\section{Figure 9. Call quality and user satisfaction with 14 VolP calls and 2Mbps background traffic (512bytes IP packets @ 488pps)}

We measured the MAC bandwidth components and user satisfaction during all experiments for 4 types of the 2Mbps background traffic. The results are presented in Figures 10-12. Figure 10 shows how the call quality decreases as the number of VoIP calls increases. Figure 11 shows how the amount of free bandwidth decreases as the number of VoIP calls increases.

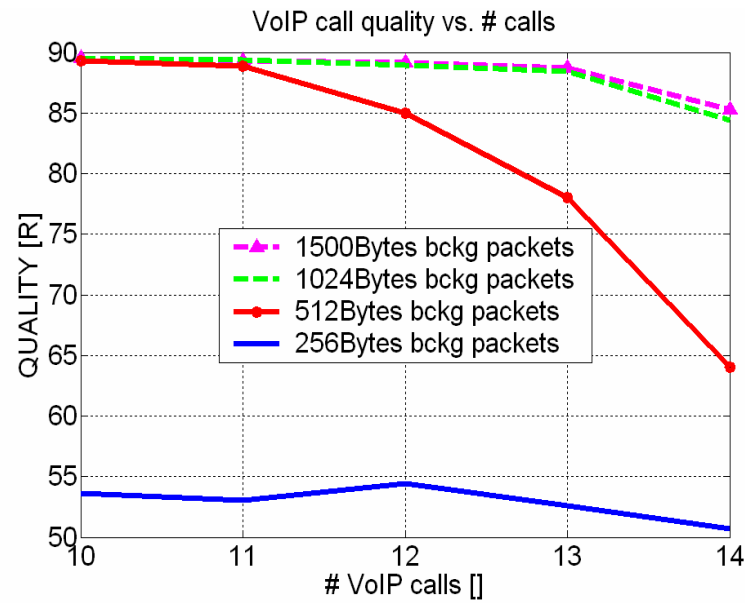

Figure 10. Call quality vs. number of VolP calls for four background traffic loads

Satisfactory call quality indicated by a rating factor $R>80$ can be achieved with maximal number of 12 voice calls with background traffic of $2 \mathrm{Mbps}$ comprised of 512Bytes IP packets. The same satisfactory call quality can be achieved for $14 \mathrm{VoIP}$ calls with the same background traffic of $2 \mathrm{Mbps}$ but with larger packets comprising the load (1024 and 1500 Bytes IP packets). When packets comprising the background load of 2Mbps are small (256 Bytes IP packets) the call quality is not acceptable even for 10 VoIP calls. Figure 11 shows how the amount of the free bandwidth decreases as number of VoIP call increases for 4 kinds of the background traffic load. It can be clearly seen that the amount of free bandwidth in the wireless network depends on the size and sending rate of the packets comprising the load.

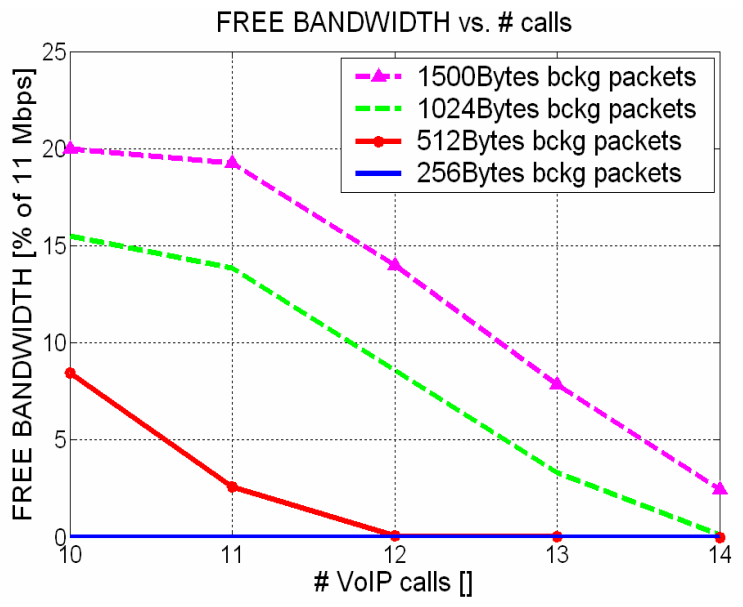

Figure 11. Amount of free bandwidth vs. number of VolP calls for four background traffic loads

Figure 12 was derived from Figures 10 and 11 and shows how the call quality depends on the availability of free bandwidth.

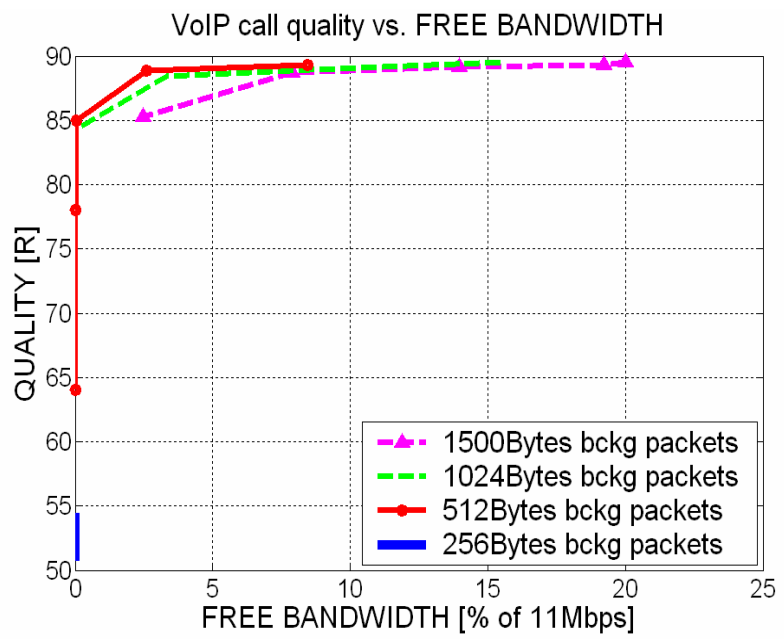

Figure 12. Call quality vs. amount of free bandwidth for two background traffic loads

As can be seen from Figure 12, a dramatic decrease in call quality can be observed when the free bandwidth falls below $1 \%$. 


\section{Conclusions}

Currently available WLAN protocol analyzers are not able to measure the bandwidth cost (i.e. the access bandwidth) associated with the time a station spends attempting to access the medium in order to transmit its load. Consequently, these analyzers cannot measure the bandwidth usage at the L2/MAC layer which makes performance monitoring and servicing provisioning on WLANs difficult to realize. The WLAN resource monitoring application passively "sniffs" packets at the L2/MAC layer of the wireless medium and provides information that characterize the resource usage in terms of three MAC bandwidth components (load, access, and free bandwidth). Of particular interest here is the ability to measure the free bandwidth which represents the spare or available capacity directly related to QoS offered to the station.

Through experimentation with a number of VoIP calls and background traffic loads in an $802.11 \mathrm{~b}$ WLAN we found a close relationship between the free bandwidth and call quality. When the amount of free bandwidth in the wireless network dropped below $1 \%$ call quality became unacceptable for all ongoing calls. We believe that this kind of information in terms of MAC bandwidth components may be required for potential QoS provisioning and call admission schemes.

Another experimental finding is that for the same level of background traffic and for the same number of VoIP calls, the quality of ongoing calls depends largely on the nature of the background load. For example when large background packets were transmitted over the wireless network with a small sending rate (1500 bytes packets at $167 \mathrm{pps}$ ), more free bandwidth was available to the VoIP stations and the quality of ongoing calls was good. Consequently it was possible to establish 14 good quality VoIP calls in the presence of $2 \mathrm{Mbps}$ of background traffic. On the other hand when small packets were transmitted with a high sending rate ( 256 bytes packet at $976 \mathrm{pps}$ ), there was no free bandwidth availabe to the VoIP stations and quality of ongoing calls was not acceptable even when only 10 VoIP calls were established. In wireless networks such as $802.11 \mathrm{~b}$ WLANs, different stations will experience different capacities that depend on the nature of the overall network load.

\section{Acknowledgments}

This work was supported by Science Foundation Ireland grant 03/IN3/I396.

\section{References}

1. M. Davis, "A Wireless Traffic Probe for Radio Resource Management and QoS Provisioning in IEEE 802.11 WLANs", ACM Symposium on Modeling, Analysis and Simlulation of Wireless and Mobile Systems (ACM MSWiM 2004), Venice, October, 2004.

2. M. Davis and T. Raimondi, "A Novel Framework for Radio Resource Management in IEEE 802.11 Wireless LANs", Intl. Symposium on Modeling and Optimization in Mobile, Ad Hoc, and Wireless Networks (WiOpt 2005), Trentino, April, 2005.

3. N. O. Johannesson: "The ETSI Computation Model: A Tool for Transmission Planning of Telephone Networks", IEEE Communications Magazine, pp. 7079, January 1997.

4. ITU-T Rec. G.107, "The E-Model, a computational model for use in transmission planning", March 2003

5. Telecommunications Industry Association, "Voice Quality Recommendations for IP Telephony TIA/EIA/TSB116", 2001

6. ITU-T Recommendation G.109, "Definition of categories of speech transmission quality", September 1999

7. Miroslaw Narbutt, Andrew Kelly, Liam Murphy, Philip Perry, "Adaptive VoIP Playout Scheduling: Assessing User Satisfaction,"IEEE Internet Computing Magazine, vol. 09, no. 4, July/August 2005.

8. Miroslaw Narbutt, Mark Davis, "Assessing the Quality of VoIP Transmission Affected by Playout Buffer Scheme," Proc. of the ETSI/IEE Measurement of Speach and Audio Quality in Networks Conference 2005 (MESAQIN 2005), Prague, June 2005.

9. Miroslaw Narbutt, Mark Davis, "An Assessment of the Audio Codec Performance in Voice over WLAN (VoWLAN) Systems," Proc. of the International Conference on Mobile and Ubiquitous Systems: Networking and Services, (MOBIQUITOUS 2005), , San Diego, July 2005.

10. Miroslaw Narbutt, Mark Davis "Gauging VoIP Call Quality from $802.11 \mathrm{~b}$ Resource Usage", Proc of the IEEE International Symposium on a World of Wireless, Mobile and Multimedia Networks (WoWMoM06), Buffalo-NY, June 2006

11. Miroslaw Narbutt, Mark Davis, "Effect of Free Bandwith on VoIP Performance in 802.11b WLANs", Proc of the IEE Irish Signals and Systems Conference 2006 (ISSC 2006), Dublin, June 2006

12. ITU-T TR 43(WP 2/12) "The E-model based quality contours for predicting speech transmission quality and user satisfaction", Geneva, Switzerland, June 2006

13. RTPtools: www.cs.columbia.edu/IRT/software/rtptools

14. ITU-T Recommendation P.59, “Artificial conversational speech", March 1993.

15. MGEN, The Multi-Generator Toolset: http://pf.itd.nrl.navy.mil/mgen/

16. R. Ramjee, J. Kurose, D. Towsley, and H. Schulzrinne, "Adaptive playout mechanisms for packetized audio applications in wide-area networks", Proc. of the IEEE INFOCOM, Toronto, 1999 